\title{
Maximizing the Output Power of Wind-Driven Grid-Connected Cage Induction Generator through Pole and Voltage Control
}

\author{
M. A. Abdel-halim* and A. F. Almarshoud \\ College of Engineering, Qassim University, Buraidah, Saudi Arabia; \\ masamie@qec.edu.sa,dr_almarshoud@qec.edu.sa
}

\begin{abstract}
Objectives: A new cheap wind-driven grid-connected cage induction generator has been used to generate electrical power. The performance of the generator has been ameliorated through multiple change of its number of poles using pole amplitude modulation to comply with the wind speed. The generator stator voltage has been adjusted via voltage controller for better performance. Methodology: Circuit and mathematical models have been developed to represent the generator system. An algorithm has been developed and implemented through computer programs to determine the performance characteristics of the turbine-generator system. The performance has been judged by comparing it by the performance when feeding the generator through a rectifier-inverter set in the stator side, which is costly but leads to a generator with ideal performance. Findings: Complete performance characteristics of the new proposed system have been computed. The obtained results have been used to establish a control protocol to guarantee maximizing the output electrical power. The suggested method has been with those of the case of using a rectifier-inverter set in the stator side to achieve ideal operation of the generator. The comparison has proved that the suggested method, being cheap, is very efficient as the proposed technique led to driving the generator and consequently the wind-turbine at speeds close or equal to those realizing the maximizing tip-speed ratio, which results in extracting the greatest mechanical energy from the wind. The output power applying the presented method closely approaches the output power of the generator when ideally controlled by the aid of a rectifier-inverter set inserted in the stator side. Applications: The very good performance obtained with the suggested control technique besides its low cost encourages its application in case of small and moderate size wind-driven grid-connected induction generator systems.
\end{abstract}

Keywords: Induction Generator, Pole Amplitude Modulation, Wind Energy

\section{List of Symbols:}

$\mathrm{C}_{\mathrm{p}}$ : Wind power coefficient

$\mathrm{C}_{\mathrm{Q}}$ : Wind torque coefficient

$\mathrm{I}_{\mathrm{r}}:$ Rotor current

$\mathrm{I}_{s}$ : Stator current

Vs: Stator voltage

$V_{\mathrm{w}}:$ Wind speed

$\mathrm{R}_{\mathrm{r}}$ : Rotor voltage

$\mathrm{R}_{\mathrm{s}}$ : Stator voltage

$\mathrm{R}_{c}$ : Core resistance

$\mathrm{R}_{\mathrm{T}}$ : Thevini's equivalence resistance

$R_{\mathrm{b}}$ : Radius of the turbine blade

$\mathrm{Xr}$ : Rotor leakage reactance

$\mathrm{X}_{\mathrm{s}}$ : Stator leakage reactance
$\mathrm{X}_{\mathrm{m}}$ : Magnetizing reactance

$\mathrm{X}_{\mathrm{T}}$ : Thevini's equivalence reactance

s: Per-unit slip

$\beta$ : Blade pitch angle

$\lambda:$ Tip-speed ratio

$\omega_{\mathrm{T}}$ : Angular speed of the wind turbine

$\tau$ : Torque

\section{Introduction}

Generation of electrical energy from the renewable wind energy has become an essential goal of energy researches nowadays. The wind-turbines as prime movers have variable and unexpected velocities. If synchronous generators are

*Author for correspondence 
used, its frequency will be no-longer constant and will vary as it directly depends on the mechanical speed. Consequently, there is a need to other types of generators or unusual solutions. Use of induction generators solves the problem of this variable speed turbines ${ }^{1-3}$. Most of the research works carried out in this field aimed at maximizing the utilization of the wind energy through controlling the generator system such that the turbine rotates at speed enables catching the possible maximum wind-energy at all wind speeds ${ }^{4-10}$.

In $^{4}$ proposed a simple technique to control a Double Fed Induction Generator (DFIG) such that it will trace the maximum power available at different wind speeds. Using a rectifier-inverter set in the rotor. $\mathrm{In}^{5}$ proposed a technique for the rotor injected voltage of a DFIG such that the wind turbine traces the maximum wind energy and at the same time the stator current is kept at unity power factor. $\mathrm{In}^{6}$, proposed a system constituting of a DFIG using a controlled cycloconverter in the rotor aiming at tracing the maximum power locus. $\mathrm{In}^{7,8}$ suggested a method of controlling the induction generator such that the driving wind-turbine follows the point of maximum wind power. This is employed through dual control using a cycloconverter inserted between the stator and the supply besides resistances inserted in the rotor circuit. Other authors suggested controlling the IG from the stator side using rectifier-inverter set ${ }^{9}$ with the aim of tracing the maximum wind power points or using a cycloconverter ${ }^{10}$ to drive the generator as near as possible to these maximum points.

In general, control of the induction generators is achieved with the aid of the various power-electronics converters inserted in the rotor and/or in the stator. If power-electronics converters are inserted in the rotor side, they will be of reduced rating (about $25 \%$ of the generator rating $)^{11}$. But in this case, the induction generator should have a slip ring rotor, which is expensive compared to the cage one. To control the cheaper cage induction generator utilizing rectifier-inverter set, the converters - because of the inaccessible rotor circuit - have to be applied in the stator which is the only available side. In this case, if a rectifier-inverter set is used, its rating should be the same as that of the generator, which is again costly. To avoid such costly converters, different control techniques should be employed for the cage induction generators like altering the generator number of pole pairs.

The present study aims at increasing the output electrical power of the induction generators at the different wind speeds through differently setting the number of poles of the generator, besides controlling the applied voltage via a low-rating low-cost AC voltage controller when necessary. Pole control will be achieved via changing the machine number of poles using pole amplitude modulation technique for the stator winding ${ }^{12}$. The study will end with proposing governing strategies to get the most of the generator electrical power. The characteristics of the generator performance when employing the suggested method will be compared with these resulting if the generator is ideally controlled through rectifierinverter set inserted in the stator side to keep continuous change of the frequency to comply with the wind speed.

The research will be performed through developing a circuit model for the controlled grid-connected cage induction generator considering pole-changing and voltage control and another one for the variable voltage and frequency input for the case of feeding the generator through a rectifierinverter set. Then mathematical models will be developed for the complete system. Based on these models, computer programs have been developed to identify the performance characteristics of the generator system. Posteriorly, thorough analysis and discussion of the resulted performance of the controlled generator will be carried out.

\section{Studied System}

The system under study comprises a wind-driven gridconnected cage induction generator controlled by pole amplitude modulation technique. The generator is linked either directly to the grid or through an ac voltage controller (Figure 1).

\section{Control Methods}

To raise the system output power, it is obligatory to upsurge the power extracted by the turbine from the wind. This is attained by shifting the generator rotational speed to reacha turbine tip-speed ratio amounting to or

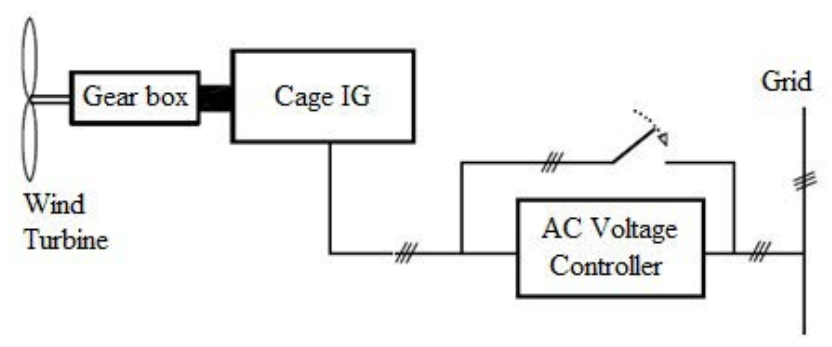

Figure 1. The wind-driven grid-connected induction generator system. 
very near to the optimum value ${ }^{13,14}$. 'Pole amplitude modulation' technique ${ }^{12}$ will be used to adjust the generator speed at 3 levels. This is performed to enable the turbine to haul out the maximum wind power by adapting its tipspeed ratio to be close to its optimum value. Moreover, the stator terminal voltage is adjusted through an AC voltage controller ${ }^{15}$ to have a value of this tip-speed ratio very near to the optimal value.

\subsection{Pole Amplitude Modulation}

The winding of the machine is apportioned to many sections in which the current is directed in one section reversely to the direction in the other. This technique adopts the principle of amplitude modulation to the magnetic motive force space distribution resulted by the windings ${ }^{12}$.

In two-sets of poles using PAM technique, the three phase windings are altered from star connected phases, where each phase has two parallel circuits, to delta connected phases, where each phase circuits are connected in series. In case of three-sets of poles, three speeds can be obtained by switching from star connected phases having 4-parallel circuits per each phase to star connected phases

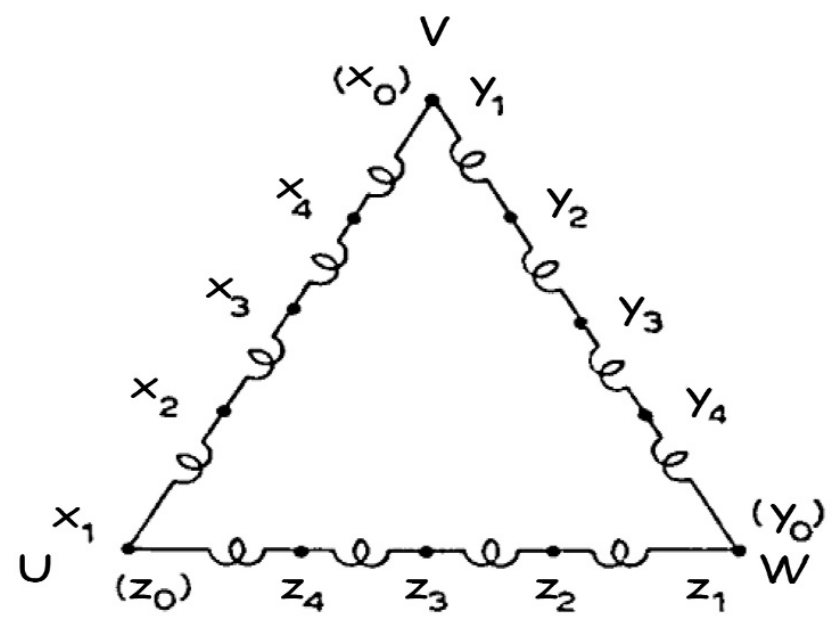

Figure 2. Circuit connection for the pole-amplitude modulation technique in case of three-speed settings. of 2-parallel circuits per phase to delta connected phases of series circuits per phase ${ }^{12}$.

With the aid of the circuit displayed in (Figure 2), the suggested connection of terminals is shown in (Table 1) to obtain certain number of poles which gives the required specific speed.

\subsection{Stator Voltage Control}

If the generator number of poles is altered, operation of the generator is shifted to a speed which is around the optimum speed. When the stator terminal voltage is decreased, a chance to adjust the speed such that $\lambda$ realizes the condition of getting the maximum power point (Figure 3) 3,17. Maximization of the generator input is expected to increase the output electrical power. It is worth to mention here that when the terminal voltage is lowered, the current should be observed and the process of voltage reduction is performed provided that the current is kept within the rated values. In other words, voltage reduction is performed as long as it leads to increase in the output electrical power, while the generator currents are bordered by the rated values.

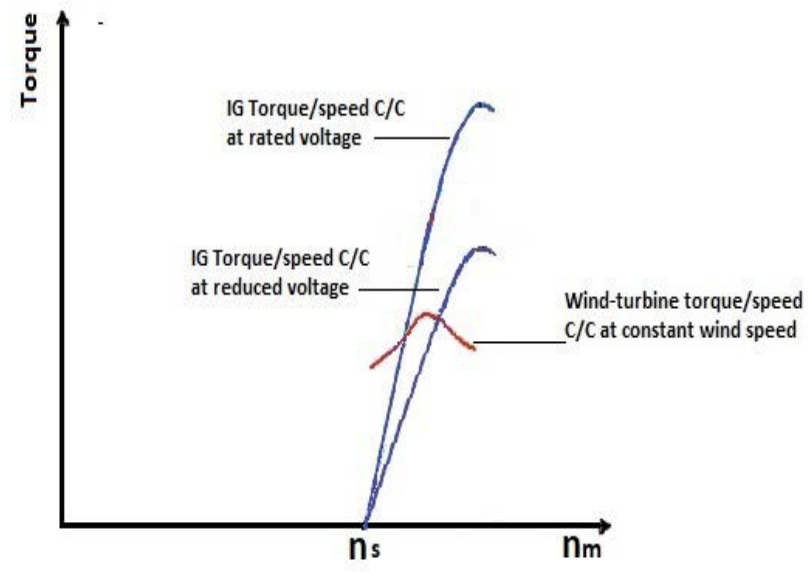

Figure 3. Tracing the maximum power point by decreasing the stator voltage.

Table 1. Connections and feeding arrangements for three-speed pole-amplitude-modulation method

\begin{tabular}{|l|l|l|}
\hline $\begin{array}{l}\text { First Speed: (four-Parallel phase- circuit with } \\
\text { 3-phase } \mathrm{Y} \text { ) }\end{array}$ & $\begin{array}{l}\text { Second Speed: (two-Parallel phase- circuit } \\
\text { with 3-phases Y) }\end{array}$ & $\begin{array}{l}\text { Speed 3: (Series phase circuit with } \\
\text { 3-phase } \Delta \text { ) }\end{array}$ \\
\hline $\begin{array}{l}\text { Connect } \mathrm{x}_{1}, \mathrm{y}_{1} \& \mathrm{z}_{1} \\
\text { Connect } \mathrm{x}_{3}, \mathrm{y}_{3} \& \mathrm{z}_{3}\end{array}$ & $\begin{array}{l}\text { Join } \mathrm{x}_{1}, \mathrm{y}_{1} \text { and } \mathrm{z}_{1} \\
\mathrm{x}_{2}, \mathrm{x}_{4}, \mathrm{y}_{2}, \mathrm{y}_{4}, \mathrm{z}_{2} \& \mathrm{z}_{4} \text { are left open }\end{array}$ & open \\
Connect $\mathrm{x}_{2}, \mathrm{x}_{4}, \mathrm{y}_{2}, \mathrm{y}_{4}, \mathrm{y}_{3} \& \mathrm{y}_{3}, \mathrm{y}_{4}, \mathrm{z}_{2}, \mathrm{z}_{3} \& \mathrm{z}_{4}$ are left \\
Feed $\mathrm{x}_{2} \mathrm{x}_{4}, \mathrm{y}_{2}, \mathrm{y}_{4}, \mathrm{z}_{2} \& \mathrm{z}_{4}$ & Feed $\mathrm{x}_{3}, \mathrm{y}_{3}$ and $\mathrm{z}_{3}$ & Feed $\mathrm{x}_{1}, \mathrm{y}_{1} \& \mathrm{z}_{1}$ \\
\hline
\end{tabular}




\section{Mechanical Side Modelling}

\subsection{Wind Turbine Mathematical Model}

Wind turbines are equipment specially designed to convert part of the wind stream energy into usable mechanical energy. Wind turbines are either vertical-axis or horizontal-axis shafts ${ }^{18}$. Nowadays, almost all commercial wind turbines used in conjunction with generators to deliver electrical power to grids have horizontal-axis twobladed or three-bladed rotors.

The torque and power are usually expressed in terms of non-dimensional torque $\left(\mathrm{C}_{\mathrm{Q}}\right)$ and power $\left(\mathrm{C}_{\mathrm{P}}\right)$ coefficients as follows ${ }^{14,15}$.

$$
\begin{aligned}
& \tau_{T}=\frac{1}{2} \rho \pi R^{3} C_{Q}(\lambda \cdot \beta) V_{W}^{2} \\
& P_{T}=\frac{1}{2} \rho \pi R^{2} C_{P}(\lambda \cdot \beta) V_{W}^{3} \\
& C_{Q}=C_{P} / \lambda
\end{aligned}
$$

Note that the two coefficients are functions of the blade pitch-angle in addition to the tip-speed ratio; $\lambda$. which is determined as follows:

$$
\lambda=\left(\omega_{\mathrm{T}} R_{\mathrm{b}}\right) / V_{\mathrm{w}}
$$

Where $\omega_{\mathrm{T}}$ is the turbine angular velocity in $\mathrm{rad} / \mathrm{s}, R_{\mathrm{b}}$ is the turbine blade radius in meter and $V_{\mathrm{w}}$ is the wind stream speed in $\mathrm{m} / \mathrm{s}$.

Figure 4 shows typical variations of $C_{\mathrm{Q}}$ and $C_{\mathrm{p}}$ for a fixed-pitch wind turbine ${ }^{15}$.

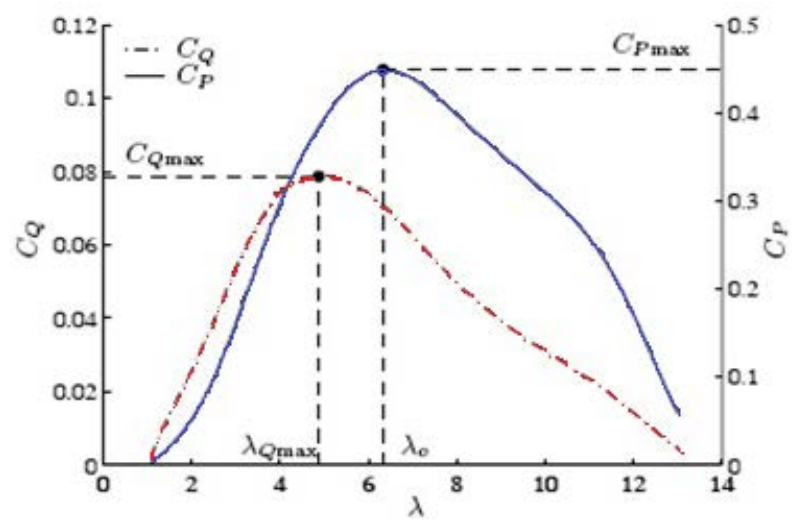

Figure 4. $\mathrm{C}_{\mathrm{Q}}$ and $\mathrm{C}_{\mathrm{P}}$ typical variation for a constant pitchangle turbine.

\section{Induction Generator Modelling}

\subsection{Pole and Voltage Controlled Generator}

Figure 5 shows steady-state circuit model of the induction generator ${ }^{3}$. The parameters of this circuit model depend on the stator circuit configuration, which in our case varies according to the generator required number of poles ${ }^{12}$.

The induced torque of the induction generator is related to applied voltage, slip and the equivalent circuit parameters as follows:

$$
\tau=\frac{3 \mathrm{~V}_{\mathrm{T}}^{2} \mathrm{R}_{\mathrm{r}} / \mathrm{s}}{\mathrm{u}_{\mathrm{s}}\left[\left(\mathrm{R}_{\mathrm{T}}+\mathrm{R}_{\mathrm{r}} / \mathrm{s}\right)^{2}+\left(\mathrm{X}_{\mathrm{T}}+\mathrm{X}_{\mathrm{r}}\right)^{2}\right]}
$$

Where $V_{\mathrm{T}}, R_{\mathrm{T}}$ and $X_{\mathrm{T}}$ are the circuit parameters when applying The venin's theorem on the stator side ${ }^{3}$.

$$
\begin{gathered}
V_{T}=\frac{\mathrm{V}_{\mathrm{s}} \mathrm{Z}_{\mathrm{ms}}}{\sqrt{\left(\mathrm{R}_{\mathrm{s}}+\mathrm{R}_{\mathrm{cs}}\right)^{2}+\left(\mathrm{X}_{\mathrm{s}}+\mathrm{X}_{\mathrm{ms}}\right)^{2}}} \\
\mathrm{R}_{\mathrm{T}}+\mathrm{j} \mathrm{X}_{\mathrm{T}}=\left(\mathrm{R}_{\mathrm{s}}+\mathrm{j} \mathrm{X}_{\mathrm{s}}\right) / / \mathrm{j} \mathrm{X}_{\mathrm{m}} / / \mathrm{R}_{\mathrm{c}}
\end{gathered}
$$

Where $\left(R_{c s}+j X_{m s}\right)$ is equivalent series impedance of the parallel components; $\mathrm{R}_{\mathrm{c}}$ and $\mathrm{X}_{\mathrm{m}}$.

\subsection{Frequency Controlled Generator}

The circuit model of Figure 5 is used in the case of feeding the generator from the variable source inverter where the reactance and slip are modified according to the frequency. The parameters will be taken corresponding to the constant used number of poles.

\section{Computation Algorithms}

A computer program has been developed to define the functioning characteristics of the studied cage induction

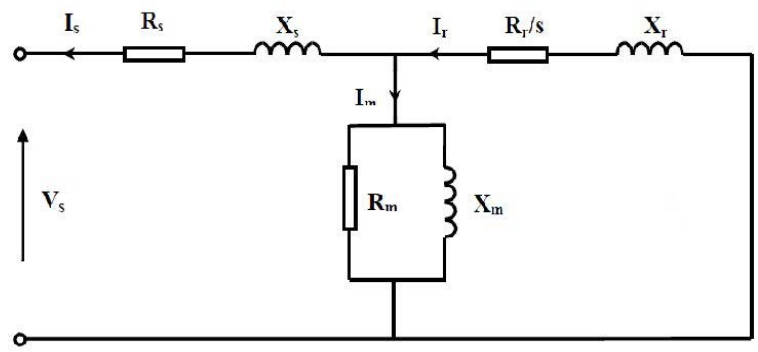

Figure 5. Per-phase equivalent circuit of the induction generator. 
generator when employing pole-modulation-amplitude and stator voltage adjustment, in case of powering the generator by wind turbines which is subjected to variable wind speeds. The computations have been based on the mathematical models given in Section 4.2.

\subsection{Pole and Voltage Control Case}

The algorithm followed during computing the generator performance for when varying the poles is,

1. At an assumed wind stream speed and number of poles, $\lambda$ is firstly calculated supposing that the generator runs at the synchronous speed using Equation 4.4. Then, the torque coefficient $\left(\mathrm{C}_{\mathrm{Q}}\right)$ is obtained from the turbine characteristic depicted in Figure 4. Consequently, the torque developed by the wind turbine is determined from Equation 4.1.

2. The P.U slip is determined from Equation 4.5. Then, the speed of the generator is recalculated. Thereafter, $\lambda, \mathrm{C}_{\mathrm{Q}}$ and the turbine torque are recalculated. The iterative technique continues till the accurate value of slip is reached.

3. The required adjusted stator voltage is calculated through the following steps:

- At the considered wind speed, the generator speed resulting inthe optimum $\lambda_{\mathrm{o}}$ which gives maximum extracted wind power is calculated. At this generator speed the corresponding slip is determined.

- $\mathrm{C}_{\mathrm{Q}}$ and the turbine driving torque is calculated at this optimum tip-speed ratio.

- The turbine torque (referred to the generator shaft) is considered the generator driving torque. Then, the venin's voltage $\left(\mathrm{V}_{\mathrm{T}}\right)$ and the stator voltage $\left(\mathrm{V}_{\mathrm{s}}\right)$ are, then, calculated using Equations 4.5 and 4.6 , respectively.

4. At the specified stator voltage (rated or reduced) and the value of the slip previously calculated, the equivalent circuit depicted in Figure 5 is used to calculate the generator currents, power factor, output power, losses etc.

5. The calculation is repeated for different wind speeds over the wind speed rang and for the three sets of poles.

\subsection{Inverter-fed Generator}

The system in this case will be adjusted such that the generator will deliver its rated VA at the maximum expected wind speed while its voltage and frequency are the rated values. Thereafter:

- At any other wind speed the generator frequency is assumed. Hence, the voltage is determined keeping $\mathrm{V} / \mathrm{f}$ constant for good performance.

- The tip-speed ratio; $\lambda$, is taken the optimum value. The generator revolving speed is determined using Equation 4.4. and the driving torque of the turbine is calculated using Equation 4.1 at the optimum $\mathrm{C}_{\mathrm{Q}}$.

- The slip is calculated and, by the aid of Equation 4.5 , the generator torque is determined.

- The turbine torque is compared to the generator torque and according to the sign of the difference, the frequency is either incrementally increased or decreased.

- The iterative technique continues till the accurate value of frequency is reached.

\section{Results and Discussion}

The developed computer program has been applied on a generator of the data and parameters given in Appendix 1. The performance characteristics at different number of poles, namely; 4, 6 and 8 are depicted in Figures 6-9.

The mechanical power captured from the wind streams clearly depends on the number of poles as shown in Figure 5. As the wind speed goes down, the number of poles ought to be increased to extract more mechanical power from the wind. For the studied generator, for speeds higher than about $9.2 \mathrm{~m} / \mathrm{s} 4$-poles should be used, while when the generator number of poles is changed to 6-poles, more mechanical power is extracted when the wind speed ranges between $9.2 \mathrm{~m} / \mathrm{s}$ and 6.9 $\mathrm{m} / \mathrm{s}$. To extract the highest mechanical power at wind speeds lower than $6.9 \mathrm{~m} / \mathrm{s}$, the number of poles has to be changed to 8 poles. The output electrical power of the generator has a pattern very analogous to the behavior of the input mechanical power as shown in Figure 6 . When the number of poles is 4 , the output electrical power is the highest compared with that of the other number of poles for the topmost wind speed value and down to about $9.2 \mathrm{~m} / \mathrm{s}$. Over the medium range of wind speed starting from about $9.2 \mathrm{~m} / \mathrm{s}$ down to about 6.9 $\mathrm{m} / \mathrm{s}$, the generator output electrical power is the highest with 6-poles. At wind speeds lower than 9.6, that of the 8 -poles is the highest. 


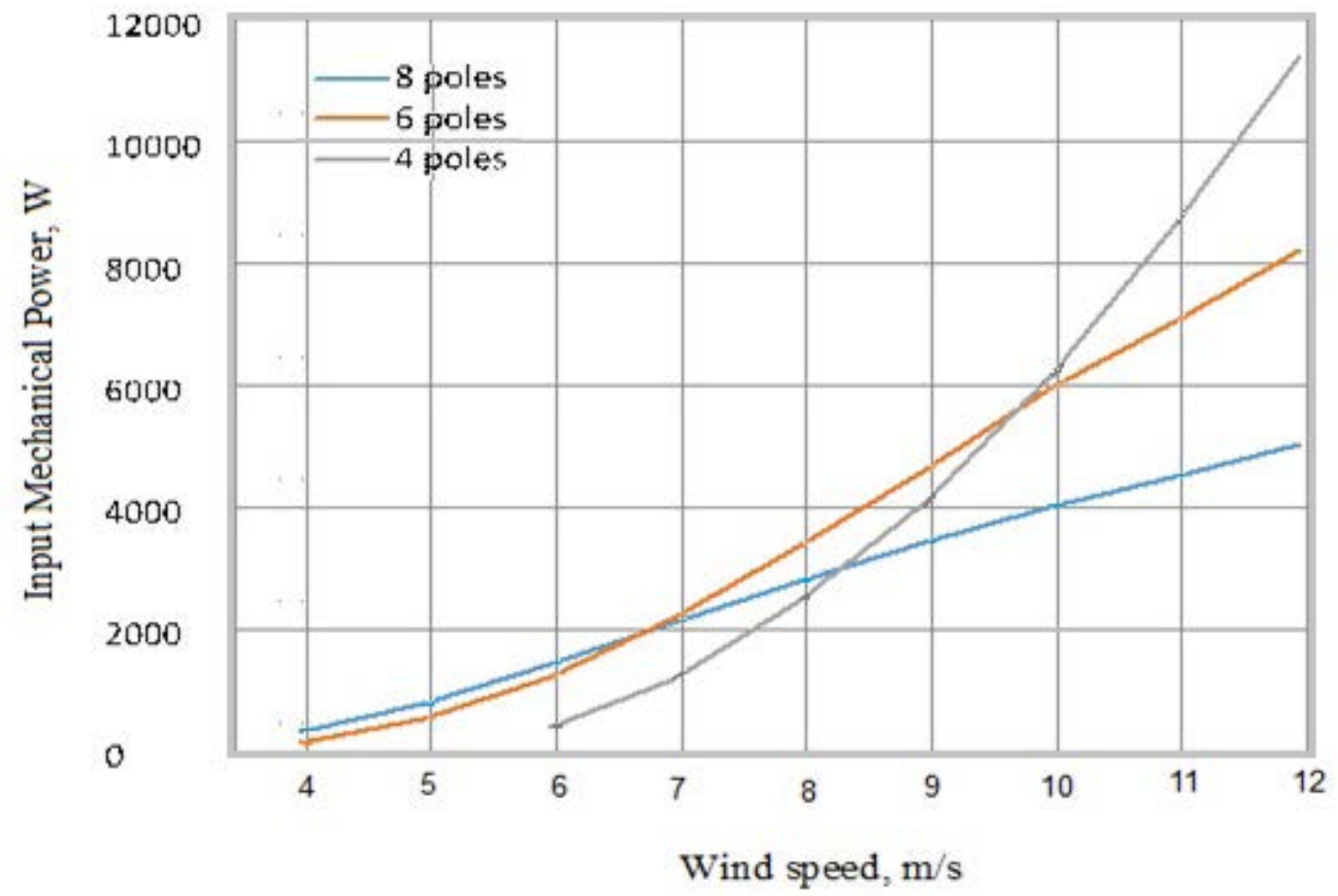

Figure 6. Variation of the input mechanical power with the wind speed for the 3 sets of poles.

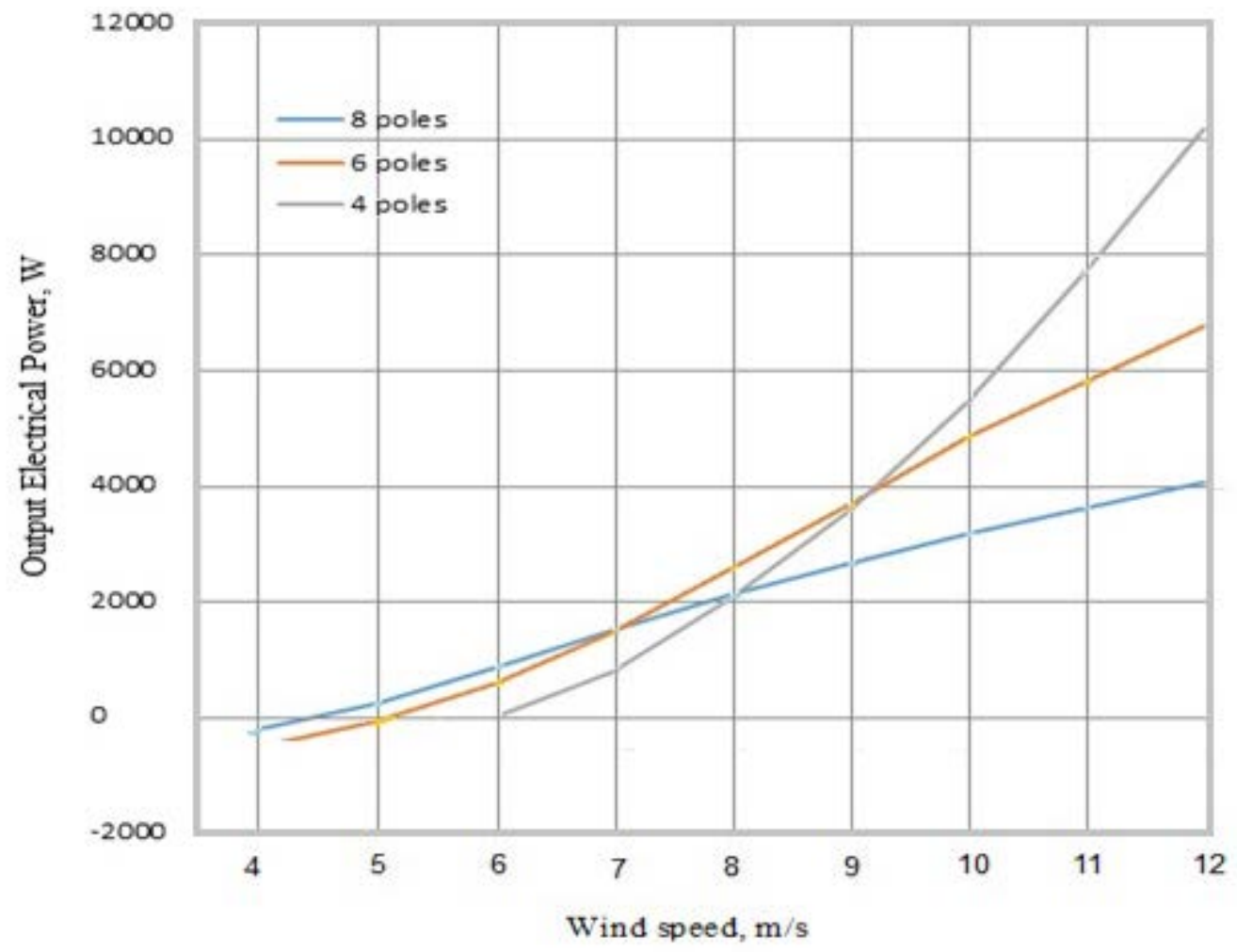

Figure 7. Variation of output electrical power with the wind speed for the 3 sets of poles. 


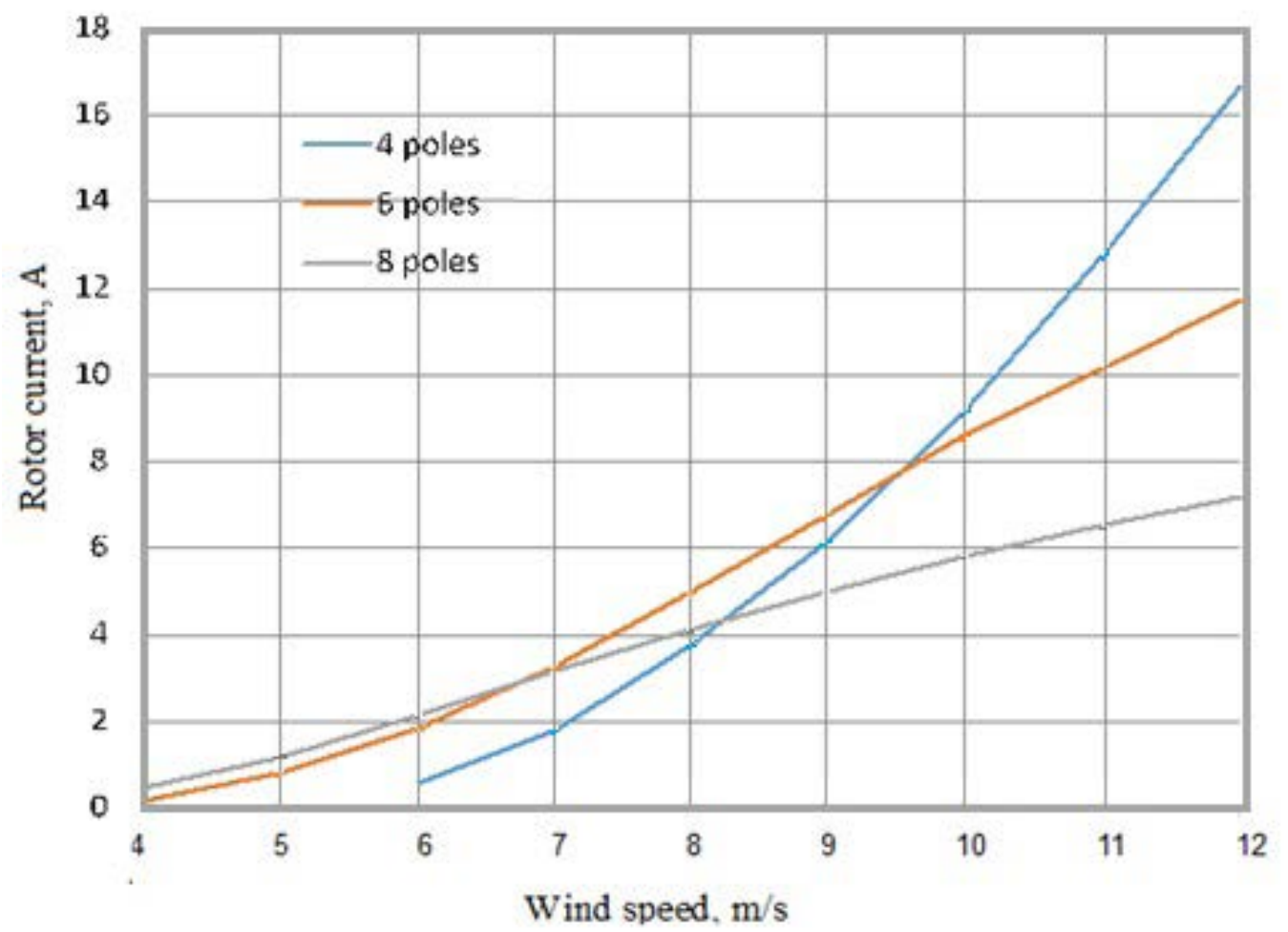

Figure 8. Variation of the rotor current with the wind speed for the 3 sets of poles.

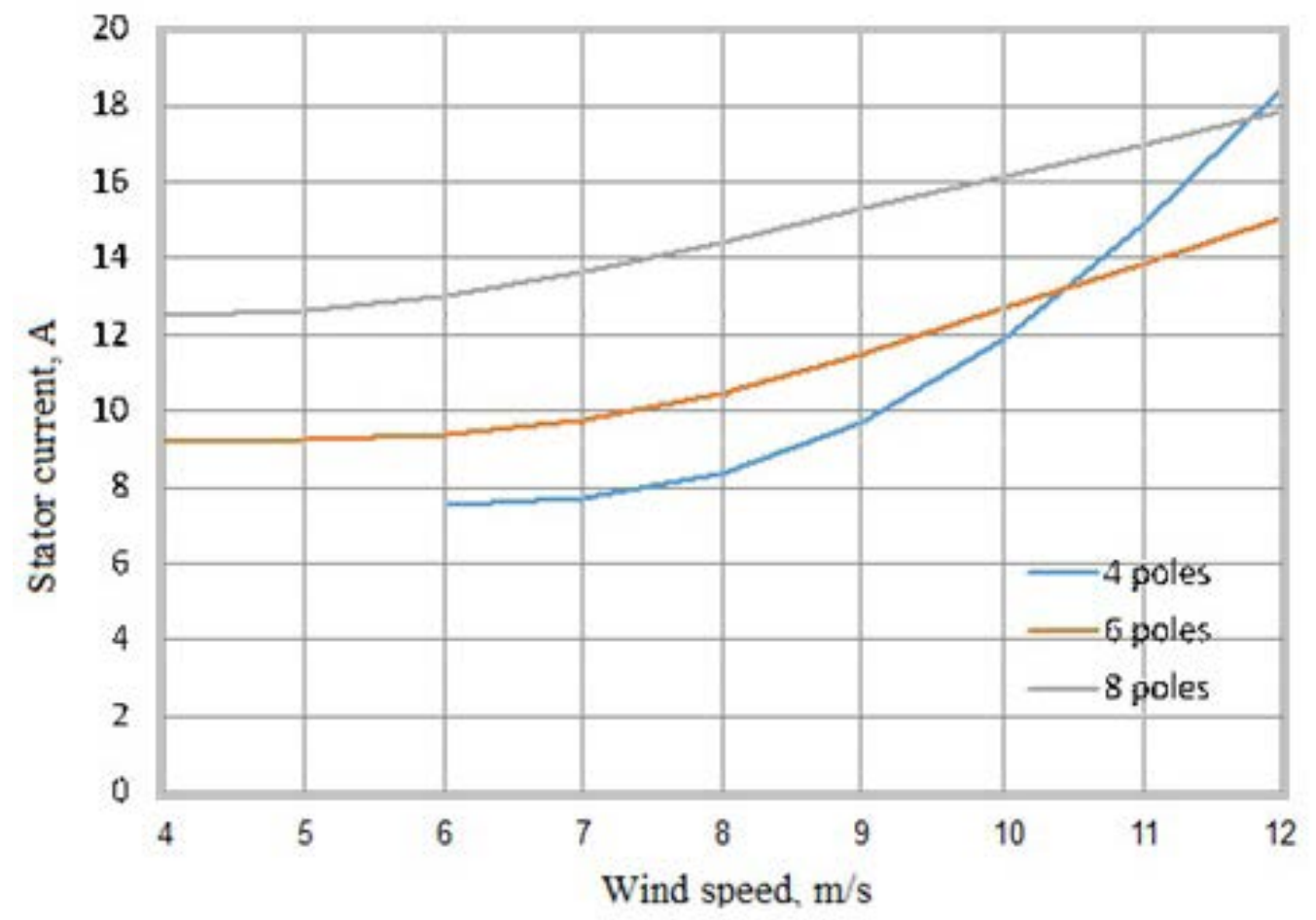

Figure 9. Variation of the stator current with the wind speed for the 3 sets of poles. 
The stator and rotor currents (Figures 7 and 8 ) decrease generally as wind speed decreases. An eye should be kept on these currents to ensure that they are within the permissible rated values. The investigation of these figures show that if the number of poles is changed corresponding to the specified wind speeds, the currents will be kept within the rated values (see Appendix 1)

The calculated power factor curves depicted in Figure 10 show that increasing the number of poles as the wind speed decreases improves the power factor, and, thus, results in less generator reactive power demand. The results of the induction generator performance at reduced voltage are shown in Figures 11-16.

Voltage reduction results have indicated that reduction of voltage is not effective at high wind speeds where the number of poles is 4 . At medium and low wind speeds somewhat increase in the output power is achieved over the lowest part of the concerned range. It can be seen from Figures 11 and 15 that the output power could be increased over specific wind speed ranges in cases of 6 and 8 poles. For 6 poles, the output power is increased over a range from about $7.9 \mathrm{~m} / \mathrm{s}$ up to about $8.4 \mathrm{~m} / \mathrm{s}$ without violating the current constraints (Figure 12). For 8 poles, the range is from about $5.87 \mathrm{~m} / \mathrm{s}$ up to about $6.2 \mathrm{~m} / \mathrm{s}$ without violating the current constraints (Figure 16). It is noted that both the stator current and voltage decrease, while the electrical power increases. This is because of the improvement of the power factor. The terminal voltage of the generator is almost halved at the upper limit of wind speed in this considered range (Figures 12 and 15). The increase of the output electrical power resulted from stator voltage adjustment is obtained through additional cost arising from the utilization of the voltage controller. Thus, benefits of the usage of the voltage controller have to be assessed against the additional cost. It is important to mention here that the rating of the suggested voltage controller will be fraction amount of the complete rating of the induction generator. In our studied generator, the voltage controller rating is about one-third of the full generator rating, as it is only applied at medium and low wind speed levels where the power dealt with is low as shown in Figures 12 and 14. Therefore, a lowrating cheap voltage controller can be inserted at low wind speeds and bypassed at high wind speeds

For comparison purposes, performance characteristics of the ideal case are depicted in Figure 16. Operation is ideal when feeding the generator from an inverter which, at a certain wind-speed, gives frequency such that the generator operates always at speed satisfying the optimum tip-speed-ratio.

The suggested cheap method when compared with the case of inverter-fed induction generator confirms its effectiveness as the output power (Figure 7) is very close to that of the ideal control method (Figure 17). If the input mechanical power employing the suggested pole control

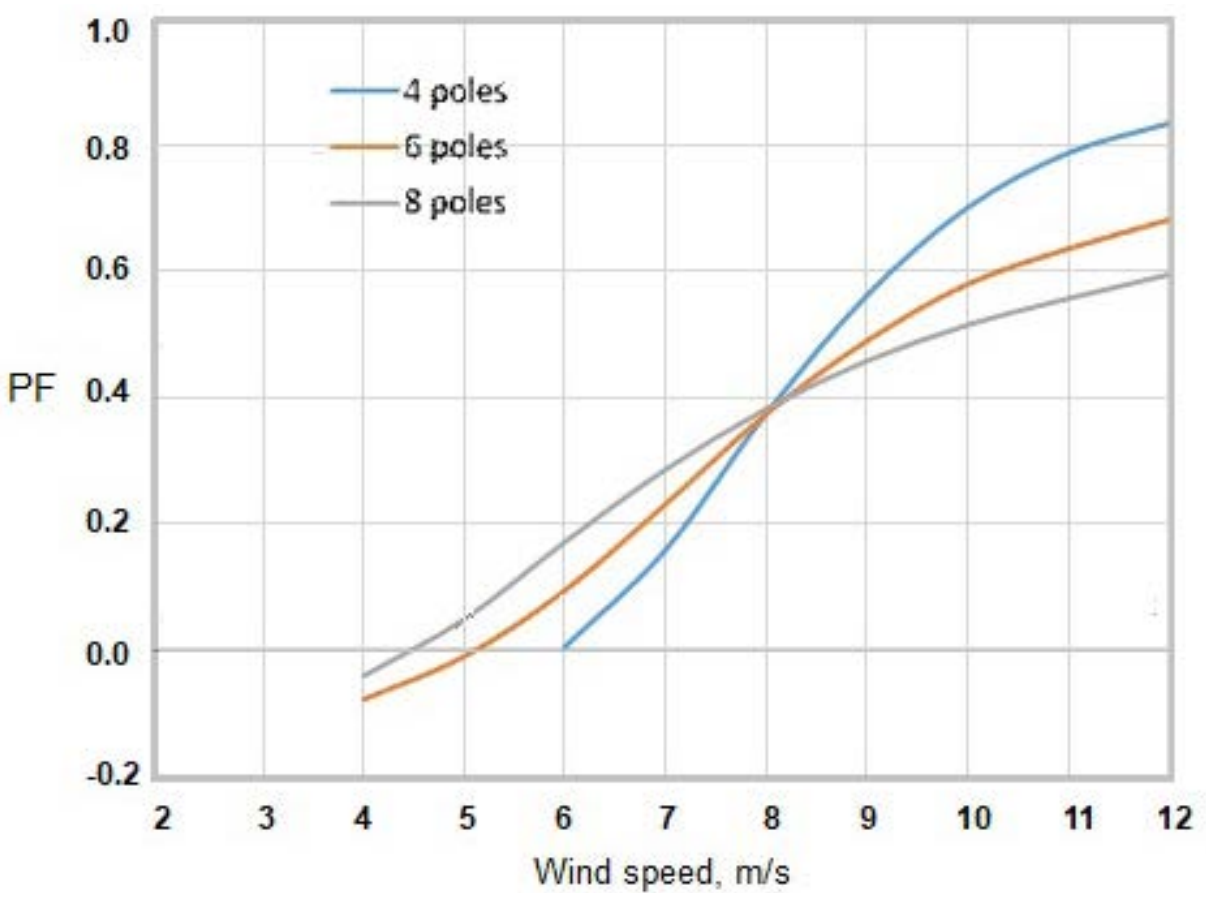

Figure 10. Variation of the power factor with the wind speed for the 3 sets of poles. 


\section{Output Power at Rated/Reduced Voltage}

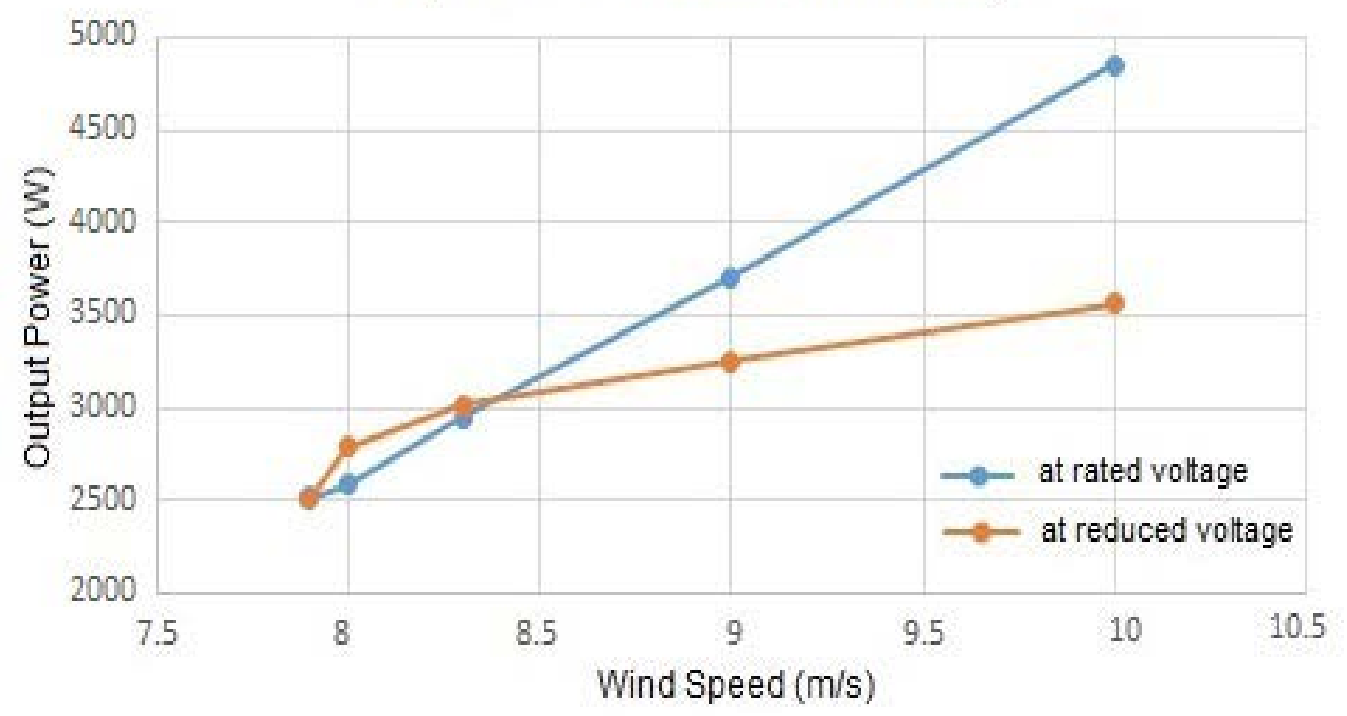

Figure 11. Effect of reducing the voltage on the output electrical power in case of 6-poles.

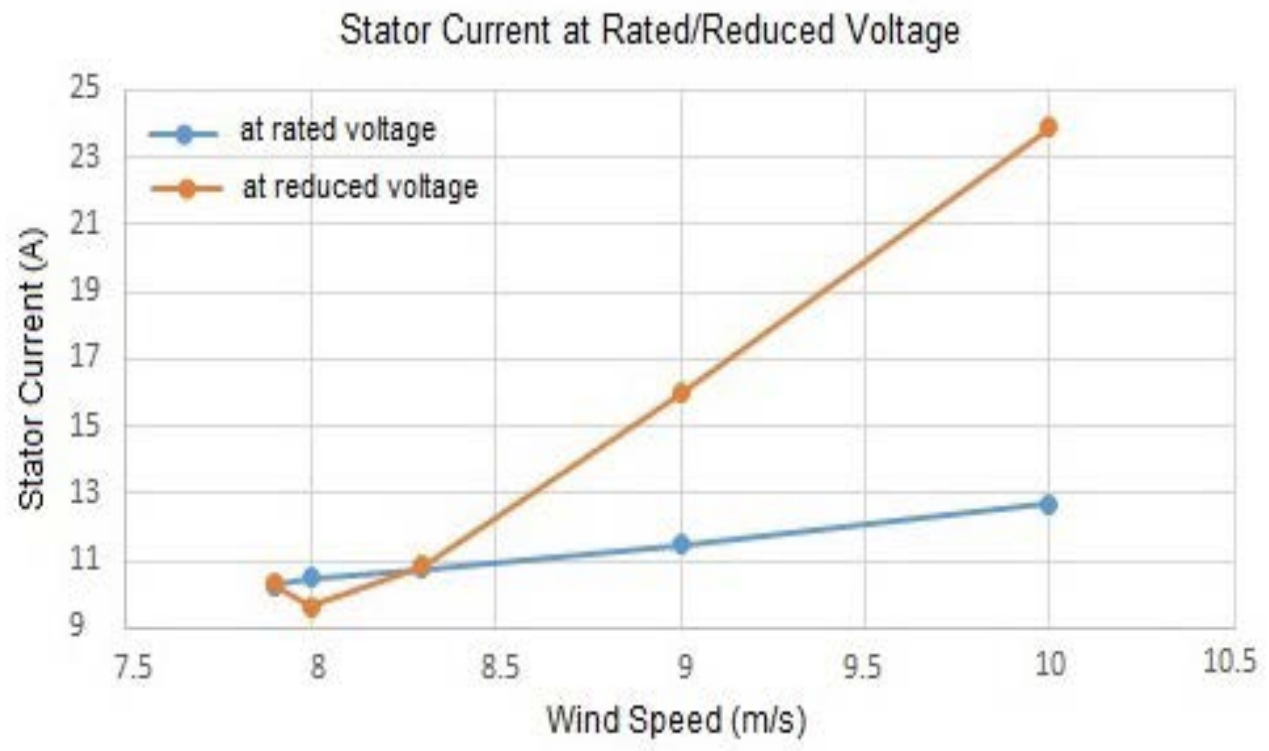

Figure 12. Effect of reducing the voltage on the stator current in case of 6-poles.

technique is compared to the ideal maximum mechanical power, it will be found that it is very close over the entire wind speed range (Figure 18).

\section{Conclusions}

Utilization of wind energy to generate electrical power is very vital. Cage induction generators- being cheap can be used in wind energy conversion system to replace the costly slip ring generators. To enhance the performance of such induction generator, pole amplitude modulation technique has been applied to increase the captured wind mechanical power and hence the output electrical power.

Pole amplitude modulation method with three sets of poles has proved its effectiveness in this regard. To cope 


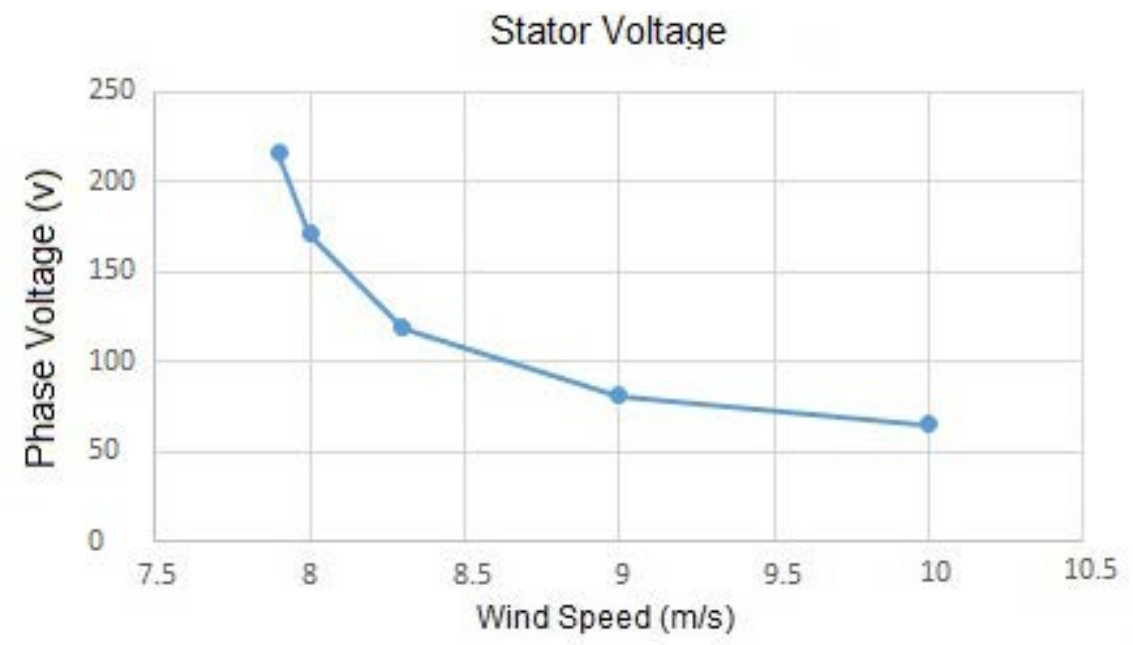

Figure 13. Stator voltage resulting in maximum input mechanical power in caseof 6-poles.

\section{Output Power at Rated/Reduced Voltage}

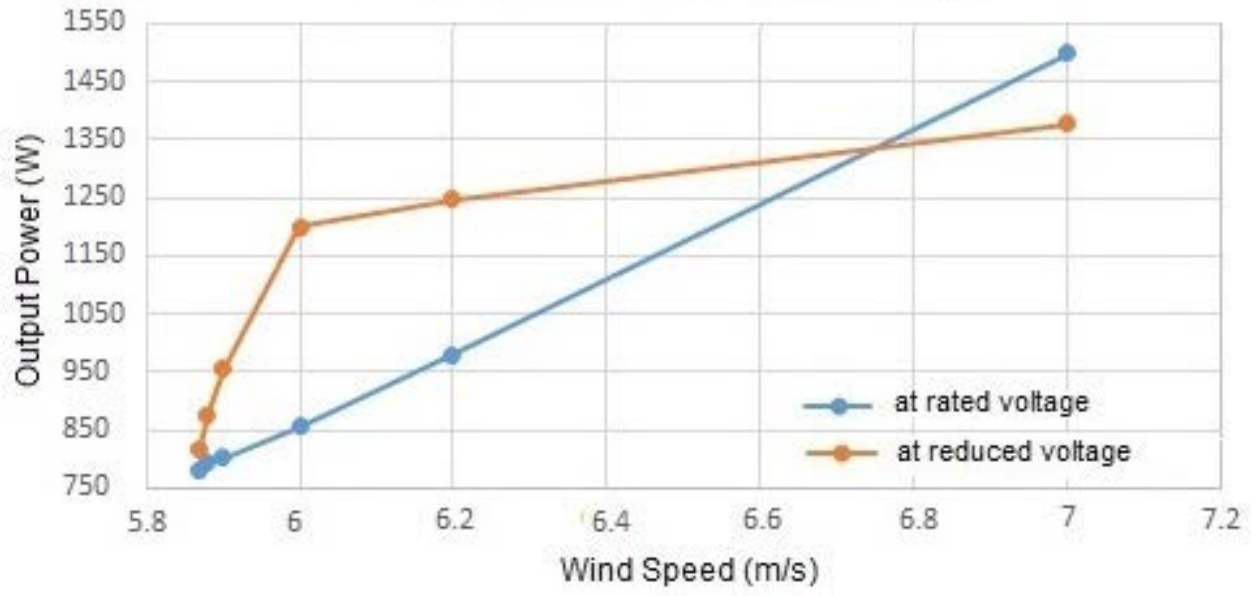

Figure 14. Effect of reducing the voltage on the output electrical power in case of 8-poles.

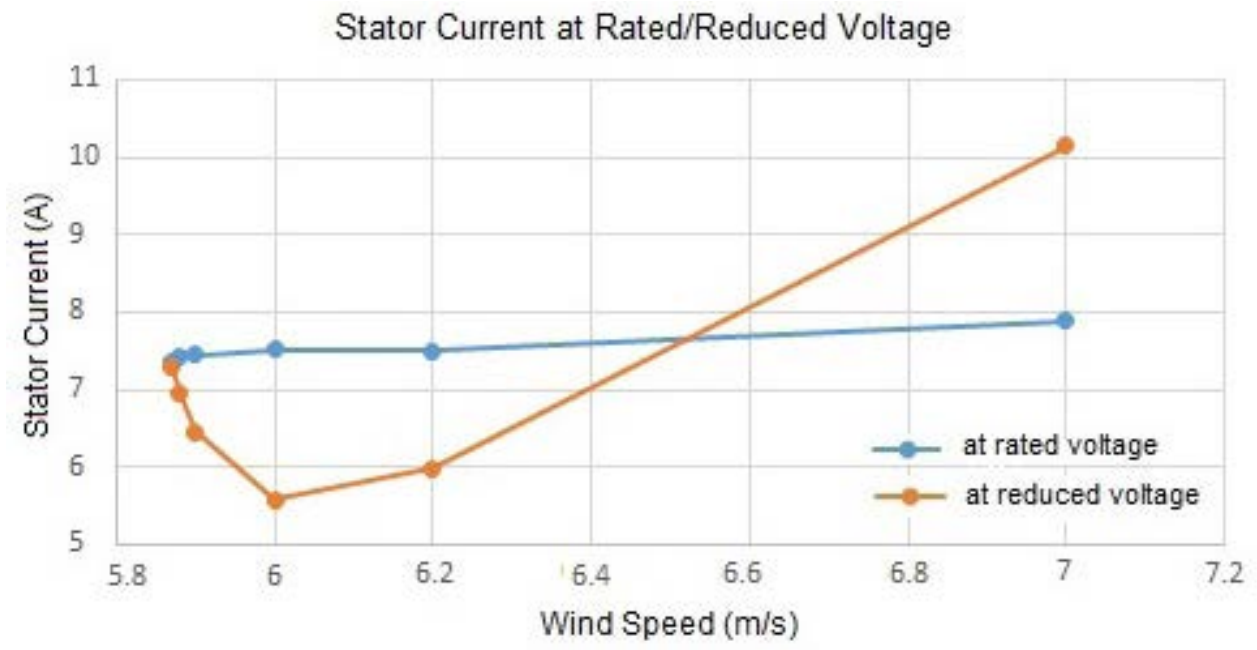

Figure 15. Effect of reducing the voltage on the stator current in case of 8-poles. 


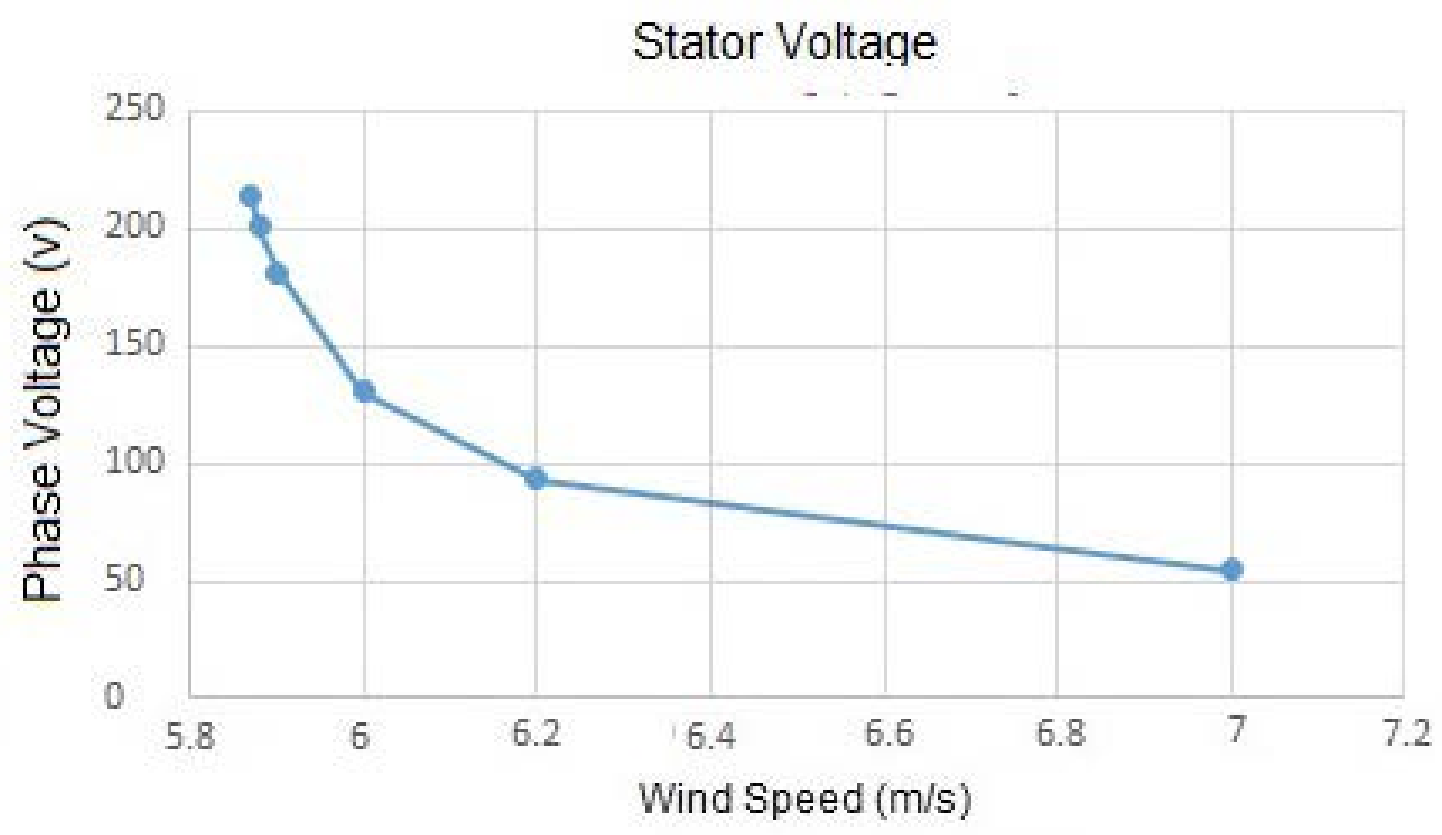

Figure 16. Stator voltage resulting in maximum input mechanical power in case of 8-poles.

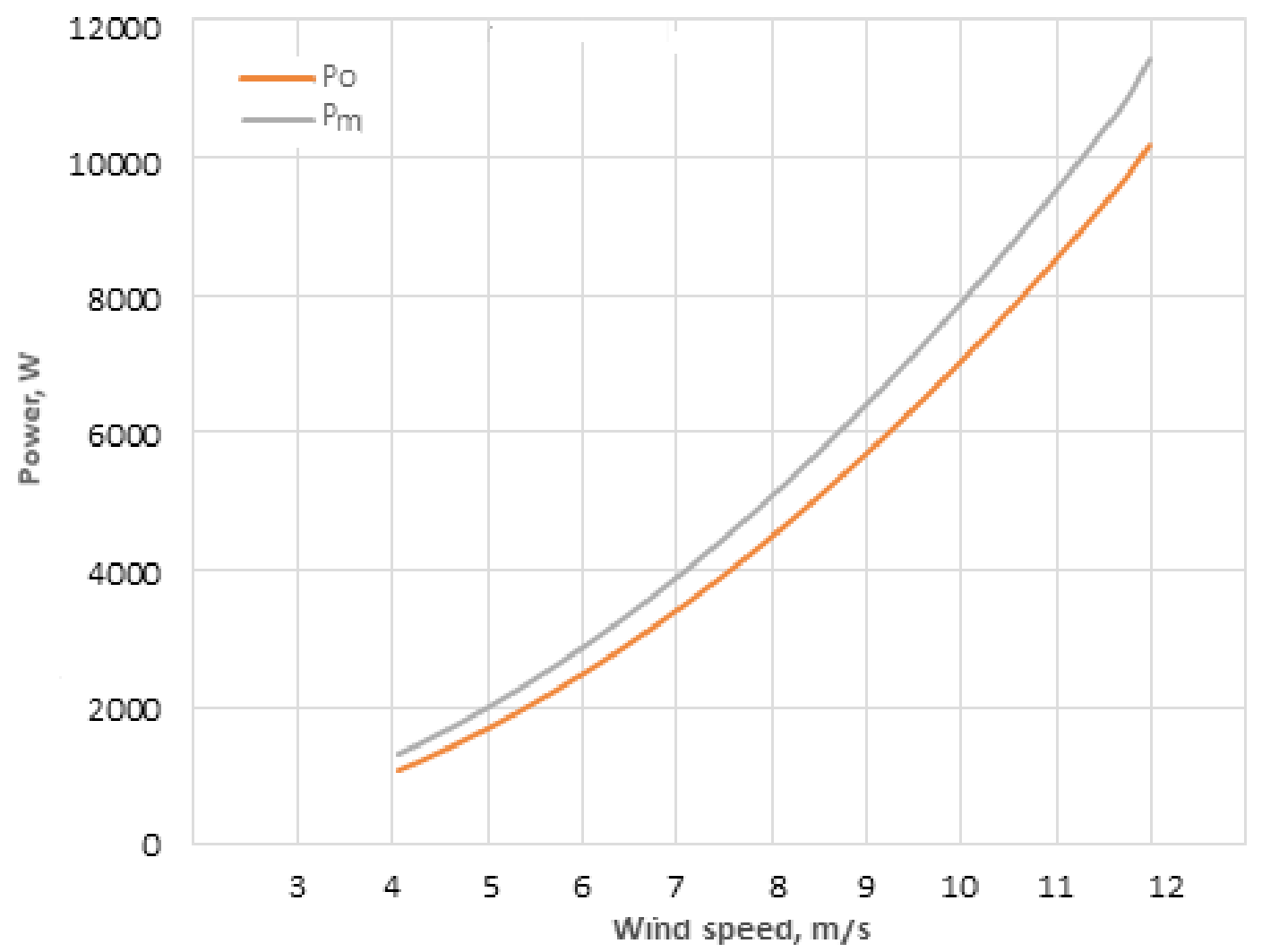

Figure 17. Input and output powers versus the wind speed. 


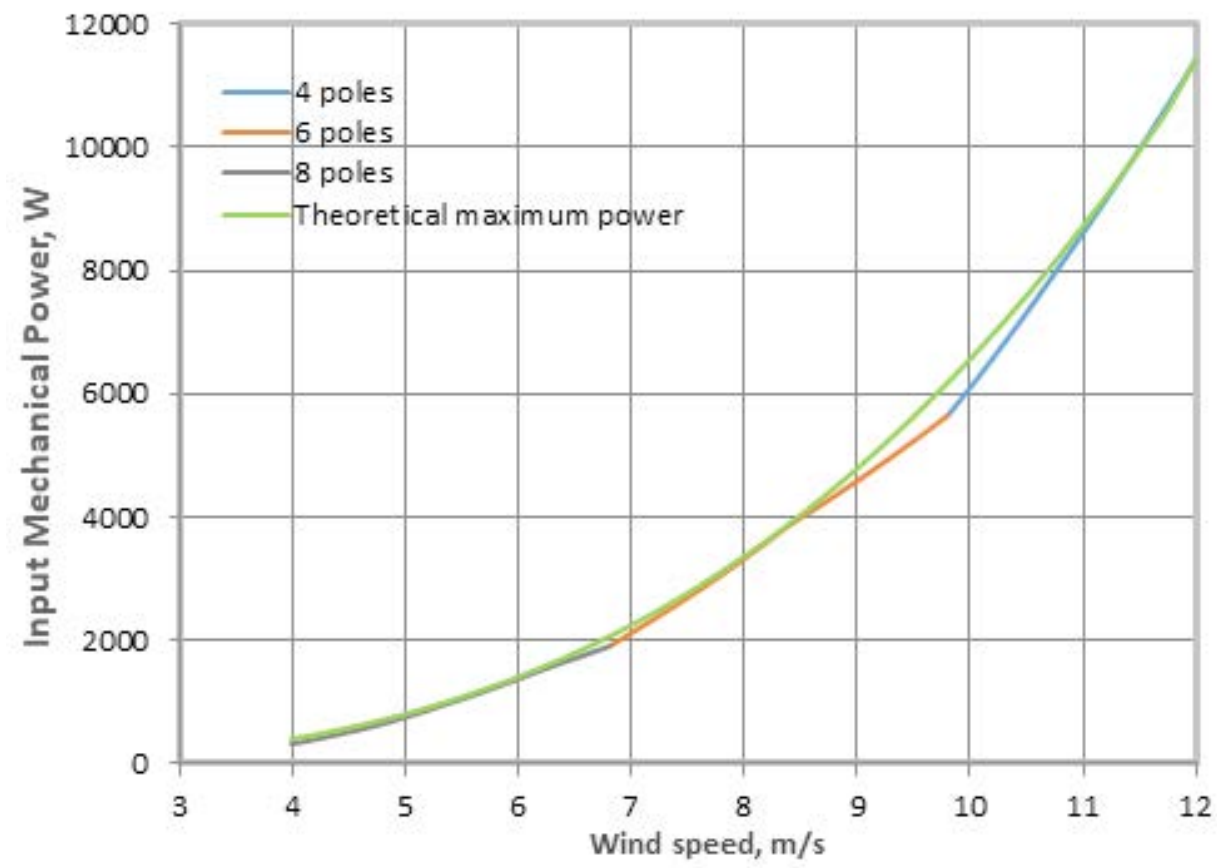

Figure 18. Input mechanical power using pole control compared with the ideal extracted maximum power.

with high wind-stream speeds, the generator stator should be switched to the least poles number. While, for medium wind-stream speeds the stator is switched to the medium poles number. For low wind-stream speeds, the stator should be switched to the highest number. Adhering to this control protocol guarantees operation of the generator and consequently, its wind turbine very near to point of maximum extracted wind-energy at all wind speeds.

In addition to altering the stator pole setting, the electrical power could be raised via stator voltage control over the low and medium ranges of wind-stream speeds. Through stator voltage reduction, the induction generator speed could be regulated so that the wind turbine follows the optimum locus of the extracted energy over divisions of the wind-stream speed range. Also, the reactive power demand of the generator has been significantly cut-down. While applying this pole and voltage control techniques, the generator currents should be observed to avoid overloading the generator. The revenue got from the more output electrical powerif stator voltage is reduced should be weighed against the extra charges of the voltage controller. The previous discussion clarified that this AC voltage controller is fortunately of low rating. Thus, its low cost justifies its use.
The suggested cheap method when compared with the case of inverter-fed induction generator confirms its effectiveness as the output power is very close to that of the ideal control method.

\section{Acknowledgment}

The authors are grateful to the Deanship of Scientific Research in Qassim University for their financial support through this research.

\section{References}

1. Singh B. Induction generators - A prospective. Electrical Machines and Power Systems. 1995; 23(2):163-77. https:// doi.org/10.1080/07313569508955615

2. Subbiah V, Geetha K. Certain investigations on a grid connected induction generator with voltage control. Proc of the IEEE International Conference on Power Electronics, Drives and Energy Systems; New Delhi, India. 1996. p. 439-44.

3. Akhmatov V. Induction generators for wind power. Essex, United Kingdom: Multi-Science Publishing Co. Ltd; 1st Ed. 2005.

4. Shaltout AA, El-Ramahi AF. Maximum power tracking for a wind driven induction generator connected to a utility network. Applied Energy. 1995; 52:243-53. https://doi. org/10.1016/0306-2619(95)00048-W 
5. Abdel-halim MA, Mahfouz AA, Almarshoud AF. Enhancing the performance of wind-energy-driven double-fed induction generators. Journal of Engineering and Computer Sciences. 2014; 7(1):23-41. https://doi. org/10.12816/0009556

6. Boumassata A, Kerdoun D, Cherfia N, Bennecib N. Performance of wind energy conversion systems using a cycloconverter to control a doubly fed induction generator. Energy Procedia. 2013; 42:143-52. https://doi. org/10.1016/j.egypro.2013.11.014

7. Abdel-halim MA. Dual control of induction generators for tracing the maximum wind energy. Journal of Engineering and Computer Sciences, Qassim University. 2015; 8(1):21-44.

8. Abdel-halim MA, Mahfouz AA, Almarshoud AF. enhancing the performance of a stator and rotor combined-controlled wind driven induction generator. Journal of King AbdulAziz University- Engineering Sciences. 2015; 26(1):3-23.

9. Variable speed generators. 2016. https://www.abebooks. com/9781498723572/Variable-Speed-Generators-SecondEdition-1498723578/plp

10. Ruben P. A cage induction generator using back to back PWM converters for variable speed grid connected wind energy system. Annual Conference of the IEEE Industrial Electronics Society. 2001;2:1376-81. PMCid: PMC1301414.

11. Abad G, Lopez J, Rodriguez MA, Marroyo L, Iwanski G. Doubly fed induction machine- modeling and control for wind energy generation. 2011. https://ieeexplore.iee.org/ book/6047757

12. Chandrasekar L, Anbuchandran S, Sankar R. Performance analysis of pole amplitude modulated three phase squirrelcage induction motor. International Journal of Emerging Technology in Computer Science and Electronics. 2015; 13:611-21.

13. Bossanyi E. The design of closed loop controllers for wind turbines. Wind Energy. 2000; 3(3):149-63. https://doi. org/10.1002/we.34

14. Leithead WE, Connor B. Control of variable speed wind turbines: Design task. International Journal of Control. 2000; 73(13):1189-212. https://doi.org/10.1080/002071700417849

15. Power Electronics and Drives. 2016. https://electronicsforu. com/resources/power-electronics-and-drives

16. Fong W. Wide-ratio Two-speed single winding induction motors. Proc IEE (UK). 1965; 112(7):1335-47. https://doi. org/10.1049/piee.1965.0222

17. Shees MM, Bakhsh FH, Asghar J, Abdel-halim MA. Power factor improvement of combined input voltage and slip power control of low power wound rotor induction generator. International Journal of Engineering Research and Applications. 2011; 1(3):963-8.

18. Ackermann, T, Soder, L. An overview of wind energy-status. Renewable and Sustainable Energy Reviews. 2002; 6(12):67127. https://doi.org/10.1016/S1364-0321(02)00008-4

\section{Appendix 1: The Generator Data}

The studied cage generator has the following data and parameters:

3 -phase, 10/6.67/5 kW, $380 \mathrm{~V}, 50 \mathrm{~Hz}, 4 / 6 / 8$ pole amplitude modulated cage induction generator. The parameters of the generator are as follows:

\begin{tabular}{|l|l|l|l|}
\hline Parameters & 4 poles & 6 poles & 8 poles \\
\hline $\mathrm{R}_{1}, \Omega$ & 0.47 & 1.034 & 1.504 \\
\hline $\mathrm{R}_{2}, \Omega$ & 0.47 & 0.972 & 1.361 \\
\hline $\mathrm{X}_{1}, \Omega$ & 0.86 & 0.7568 & 0.86 \\
\hline $\mathrm{X}_{2,} \Omega$ & 0.86 & 0.7568 & 0.86 \\
\hline $\mathrm{X}_{\mathrm{m}}, \Omega$ & 28.27 & 23.129 & 29.55 \\
\hline $\mathrm{R}_{\mathrm{c}}, \Omega$ & 500 & 409.07 & 522.710 \\
\hline $\mathrm{R}_{\mathrm{T},}, \mathrm{0}$ & 0.444 & 0.968 & 1.412 \\
\hline $\mathrm{X}_{\mathrm{T}}, \Omega$ & 0.77 & 0.899 \\
\hline Stator Rated Current, A & 18.44 & 14.98 & 13.68 \\
\hline Stator Connection & Star & Star & Delta \\
\hline
\end{tabular}

